\title{
Food Refusal, Loss of Appetite, Chronic Fatigue and Depression due to Central Adrenal Insufficiency Presenting as Anorexia Nervosa in an Adolescent Girl
}

\author{
Martine KF Docx ${ }^{1 *}$, Annik Simons², José Ramet ${ }^{3}$, Luc Mertens ${ }^{4}$ and Paul Govaert ${ }^{1}$ \\ ${ }^{1}$ Department of Paediatrics, Chronic Diseases and Eating Disorders, Queen Paola Children's Hospital Antwerp, Antwerp, Belgium \\ ${ }^{2}$ Department of Child and Adolescent Psychiatry, General Hospital Middelheim and University of Antwerp, Antwerp, Belgium \\ ${ }^{3}$ Department of Paediatrics, University Hospital Antwerp, Antwerp, Belgium \\ ${ }^{4}$ Division of Cardiology, The Hospital for Sick Children, Toronto, Canada
}

\begin{abstract}
The hypothalamic- pineal region is involved in the regulation of different functions in our body like the regulation of hunger, thirst, temperature and reproduction. In one 14-year-old anorexia nervosa patient we found low levels of ghrelin in the acute stage and during the whole refeeding procedure, suggestive of an underlying endocrine problem. The refeeding process in our patient was difficult. An insulin stimulation test was done and we observed no response of ACTH and cortisol secretion to severe hypoglycemia. Central adrenal insufficiency was found and supplementation with hydrocortisone was started. Central adrenal insufficiency explained also the difficulties in the water and sodium balance following ingestion of DDAVP for enuresis nocturna and carbamazepine for an epileptic insult.
\end{abstract}

Keywords: Anorexia nervosa; Food refusal; Chronic fatigue syndrome; Depression; Central adrenal insufficiency

\section{Introduction}

The estimated prevalence of secondary and tertiary (central) adrenal insufficiency is $150-280$ per million [1]. A lot of processes that involves the hypothalamus and which interferes with CorticotrophinReleasing Hormone (CRH) secretionare hypothalamic tumors, surgery, irradiation and autoimmune hypothalamic disease will result in central adrenal failure [2]. The symptoms that we encounter in these patients are weakness, fatigue, myalgias and psychiatric symptoms and are misdiagnosed as depression and as chronic anxiety disorders [3].

\section{Case Report}

A 14-year-old girl with a restrictive type of anorexia nervosa since the age of 13 years (BMI: $12.2 \mathrm{~kg} / \mathrm{m}^{2}$ ) was admitted to our tertiary eating disorder department. Her personal history was complicated by a hemolytic-uremic syndrome at the age of 4.5 years. After recovery of this nephrologic insult, she suffered from persistent enuresis nocturna, resistant to different treatments. At the age of 10 years old she developed an epileptic insult and was treated with carbamazepine. At the age of 12 years she received for her still persistent enuresis nocturna a treatment with DDAVP. During that treatment she developed chronic sodium deficiency, macrocytic anemia and weight loss. The family history was noticeable for several episodes of depression in her mother, who took multiple medications to brake her appetite. At the time of presentation patient's oral temperature was $35.9^{\circ} \mathrm{C}$, blood pressure $75 / 46 \mathrm{~mm} \mathrm{Hg}$ taken in sitting position with an electronic device, pulse rate was 45 bpm, oxygen saturation was $99 \%$ in room air. Her weight was $26.4 \mathrm{~kg}$, height: $147 \mathrm{~cm}$ and BMI: $12.2 \mathrm{~kg} / \mathrm{m}^{2}$. On examination her heart sounds were regular but bradycardic and there were no abnormal lung sounds. Abdominal examination was normal. She had split back and face; the extremities were acrocyanotic with livido reticularis from feet to knees. The capillary refill was delayed and there was no pitting edema. Split appeared bradyphrenic and depressive. During hospitalization hemoglobin levels dropped to $6.9 \mathrm{~g} / \mathrm{dl}$ (normal: 12.1-14.6 g/dl) with increased MCV of $93.5 \mathrm{fl}$ (normal: 79.7-93.0 fl) and MCHC of $35.6 \mathrm{~g}: \mathrm{dl}$ (normal: 33.2-35.2 g:dl). There was leukopenia of $2.4110^{9} / \mathrm{L}$ (normal: 4.5-10.7 $10^{9} / \mathrm{L}$ ) with normal platelets. Clotting factors, folic acid, vitamin B12, Vitamin E and serum iron were normal. Ferritin (3.5 ng/ $\mathrm{ml}$ ) was low with normal transferrin and decreased iron saturation of 16\% (normal: $20-50 \%$ ). Because we discovered a macrocytic anemia, a bone marrow aspirate was done and revealed normal cellularity with a reactive bone marrow and iron depletion. Patient's biochemical data yielded a hyponatriemia of $128 \mathrm{meq} / \mathrm{L}$ (135-145 meq/L) with increased natriuresis (levels between 94-341) and a hyperosmolarity of the urine of 550 till 1283. 24 Hours urine collection of aldosteron was low: 1820 $\mathrm{ng} / 24 \mathrm{hr}$ (normal diet: 6000-25000 ng/ml). FT4 and FT3 levels were low. IGF-1 was $63 \mathrm{ng} / \mathrm{ml}$ (normal: 190-716 ng/ml). The level of growth hormone was $18.00 \mathrm{ng} / \mathrm{ml}$ (normal: $0.14-11.7 \mathrm{ng} / \mathrm{ml}$ ) and of cortisol: $29.80 \mu \mathrm{U} / \mathrm{dl}$ (normal: $4.5-22.7 \mu \mathrm{U} / \mathrm{dl}$ ), both were increased. MRI of the hypothalamopituitary area and brain were normal. Bone age was 12 years. Electroencephalogram showed bilateral diffuse slow waves. Cardiac and electrocardiographic measurements in the acute stage: QTc dispersion: $48 \mathrm{msec}$; QT interval: $387 \mathrm{msec}$ Q QTc interval: 368 msec and LVM: $64.34 \mathrm{~g}$; LVM: height ${ }^{2.7}: 25.57 \mathrm{~g} / \mathrm{m}^{2.7}$. The refeeding process in our patient was difficult. The patient never had a feeling of hunger, suffered from a chronic fatigue syndrome and developed in the follow-up several episodes of sudden hyponatriemia. An insulin stimulation test was done and we observed no response of ACTH and cortisol excretion to severe hypoglycemia (Table 1). Central adrenal insufficiency was found and supplementation with hydrocortisone was started. Afterwards her symptoms ameliorated and disappeared after nearly one year.

*Corresponding author: Martine KF Docx, Queen Paola Children's Hospital Antwerp, Lindendreef 12020 Antwerp, Belgium, Tel: (32)-3- 2803341; Fax: (32) 3-2802133; E-mail: martine.docx@zna.be

Received April 16, 2013; Accepted October 31, 2013; Published November 02 2013

Citation: Docx MKF, Simons A, Ramet J, Mertens L, Govaert P (2013) Food Refusal, Loss of Appetite, Chronic Fatigue and Depression due to Central Adrena Insufficiency Presenting as Anorexia Nervosa in an Adolescent Girl. J Clin Case Rep 3: 310. doi:10.4172/2165-7920.1000310

Copyright: @ 2013 Docx MKF, et al. This is an open-access article distributed under the terms of the Creative Commons Attribution License, which permits unrestricted use, distribution, and reproduction in any medium, provided the original author and source are credited. 


\begin{tabular}{|c|c|c|c|c|}
\hline Time (min) & Glycemia (mg/dl) & ACTH $\mathbf{( p g / m l )}$ & Cortisol $(\boldsymbol{\mu g} / \mathbf{d l})$ & $\mathbf{G H}(\mathbf{n g} / \mathbf{m l})$ \\
\hline 0 & 80 & 13 & 5.56 & 1.07 \\
\hline 20 & 25 & 13 & 3.70 & 0.73 \\
\hline
\end{tabular}

Table 1: Insulin test: no excretion of ACTH and cortisol on severe hypoglycemia.

\section{Discussion}

Adrenal insufficiency is a life-threatening condition that may result from primary adrenal failure or from impairment of the hypothalamicpituitary adrenal (HPA) axis [1-4]. Patients with central adrenal insufficiency are a diagnostic challenge [5]. The reference tests for establishing the integrity of the HPA axis rely on a response to either a strong stimulus [e.g. insulin-induced hypoglycemia/insulin tolerance test (ITT)], or the interruption of negative feedback (overnight metyrapone test). The ITT is contraindicated in infants and children with a history of seizures or cardiovascular disease and requires continuous monitoring [6].

Central adrenal insufficiency explained also the difficulties in the water and sodium balance following ingestion of DDAVP for enuresis nocturna and carbamazepine for an epileptic insult in our patient.

The reported incidence of carbamazepine-induced hyponatriemia varies between 4.8 to $40 \%$. Carbamazepine has been associated with the Syndrome of Inappropriate Antidiuretic Hormone (SIADH) secretion, due to an impaired sensitivity of hypothalamic osmoreceptors to serum osmolality and an increased renal sensitivity of the renal tubules to circulating vasopressin $[7,8]$. Carbamazepine dose and plasma levels are probably related to the risk of hyponatriemia, while the duration of the treatment does not contribute to the hyponatriemia [7-9].

Desmopressin (DDAVP) used for enuresis nocturna in our patient causes hyponatriemia in $15 \%$ of the pediatric population. Factors that predispose to hyponatremia are a higher dose, higher age ( $>65$ years of age), a low-normal serum sodium, a high 24 -hour urine volume and co-medication (thiazides, tricyclic antidepressants,serotine-reuptakeinhibitors, chlorpromazine, carbamazepine, loperamide, non-steroidalanti-inflammatory-drugs [4-10].

\section{Conclusion}

Tertiary adrenal insufficiency is due to a hypothalamic disease and decrease in Corticotropin Releasing Factor (CRF). Oxidative damage to any of the HPA-Axis organs can be the cause of "Chronic Fatigue Syndrome" with difficulties of refeeding in our patient. Therefore in these patients with anorexia with refeeding difficulties, no hunger sensation and with chronic fatigue syndrome a central adrenal insufficiency must be excluded.

\section{References}

1. Arlt W, Allolio B (2003) Adrenal insufficiency. Lancet 361: 1881-1893.

2. Endert E, Ouwehand A, Fliers E, Prummel MF, Wiersinga WM (2005) Establishment of reference values for endocrine tests. Part IV: Adrenal insufficiency. Neth J Med 63: 435-443.

3. Kaushik ML, Sharma RC (2003) Addison's disease presenting as depression. Indian J Med Sci 57: 249-251.

4. Hiroi N, Yoshihara A, Sue M, Yoshino G, Higa M (2010) Central adrenal insufficiency and diabetes insipidus misdiagnosed as severe depression. Clin Med Insights Case Rep 3: 55-58.

5. Shulman DI, Palmert MR, Kemp SF; Lawson Wilkins Drug and Therapeutics Committee (2007) Adrenal insufficiency: still a cause of morbidity and death in childhood. Pediatrics 119: e484-494.

6. Maghnie M, Strigazzi C, Tinelli C, Autelli M, Cisternino M, et al. (1999) Growth hormone $(\mathrm{GH})$ deficiency (GHD) of childhood onset: reassessment of $\mathrm{GH}$ status and evaluation of the predictive criteria for permanent GHD in young adults. J Clin Endocrinol Metab 84: 1324-1328.

7. Van Amelsvoort T, Bakshi R, Devaux CB, Schwabe S (1994) Hyponatremia associated with carbamazepine and oxcarbazepine therapy: a review. Epilepsia 35: 181-188.

8. Henry DA, Lawson DH, Reavey P, Renfrew S (1977) Hyponatraemia during carbamazepine treatment. Br Med J 1: 83-84.

9. Staikou C, Mani A, Petropoulos G (2012) Perioperative management of a parturient with hyponatraemia due to carbamazepine therapy. Int J Obstet Anesth 21: 93-97.

10. Ten Doesschate T, Reichert LJ, Claassen JA (2010) Desmopressin for nocturia in the old: an inappropriate treatment due to the high risk of side-effects? Tijdschr Gerontol Geriatr 41: 256-261. 